\title{
Transport and quantum walk of nonclassical light in coupled waveguides
}

\author{
Amit Rai, G. S. Agarwal, and J. H. H. Perk \\ Department of Physics, Oklahoma State University, Stillwater, Oklahoma 74078, USA
}

(Received 24 May 2008; published 8 October 2008)

\begin{abstract}
We study the transport and quantum walk of nonclassical light in an array of coupled waveguides which have properties like very low decoherence and thus making them ideal for storage of quantum information. We show how squeezing gets turned over from one waveguide to another. We further show how input nonclassical light can generate entanglement among different waveguides. Our results, which are valid for an arbitrary number of waveguides, involve both first quantization due to array structure and second quantization due to the quantum nature of fields and can also be used to discuss the Talbot effect in the quantum regime.
\end{abstract}

DOI: 10.1103/PhysRevA.78.042304

PACS number(s): 03.67.Bg, 42.50.Ex, 42.50.Lc, 42.50.St

Optical elements like beam splitters are known to behave quite differently when it comes to single photons. A single photon according to Dirac can either be transmitted or reflected [1]. It cannot be found simultaneously in both transmission or reflection. The behavior of a photon pair on a beam splitter is even more remarkable as shown by Hong, $\mathrm{Ou}$, and Mandel [2]. For a 50-50 beam splitter both photons are found either in transmission or in reflection. This leads to entanglement of photons at the two output ports. The question of entanglement at the output of a beam splitter was investigated in very general terms $[3,4]$. Even more remarkable behavior has been shown experimentally [5-7] and theoretically [8]. For other optical elements like phase shifters a quantum field with a precise number of photons $n$ undergoes a phase shift which is $n \phi$ whereas a classical beam undergoes a phase shift $\phi$. It is thus important to understand the behavior of single photons and more generally nonclassical light at other optical elements. Very recently coupled waveguide systems, which are relatively easy to fabricate [9] and which are also relatively decoherence free, have been shown to be good candidates for continuous time random walks [10]. The paper by Perets et al. [10] deals with classical beams of light. In the light of what we have learned with beam splitters and phase shifters and the fact that Feynman [11] used the term quantum walk to describe the behavior of quantum particles, it is worth examining how single photons and more generally nonclassical light would behave in coupled waveguide systems. This way we would be able to understand quantum walk by single photons in coupled waveguides [12-16].

In this paper we consider the system of coupled waveguides and report the propagation of single photons and nonclassical light. We discuss how nonclassical light from one waveguide gets transported to other waveguides. This is especially important in applications to quantum-information science where one is very often interested in the storage and retrieval of a quantum state $[17,18]$. We further report how nonclassical light at one input port can entangle different waveguides. We present analytical results for Heisenberg operators and wave functions for fields in different waveguides. For coupled waveguides, we show an analog of the wellknown Hong-Ou-Mandel two-photon interference. We also report the amount of squeezing that can be produced in different waveguides.
We consider an array of $N$ single-mode waveguides, coupled through nearest-neighbor interaction as in Refs. $[9,10]$ (see Fig. 1). We will number the waveguides from 1 to $N$. The mode for the field in the $j$ th waveguide is described by the annihilation (creation) operator $a_{j}\left(a_{j}^{\dagger}\right)$. The operators $a_{j}$ and $a_{j}^{\dagger}$ for the coupled waveguide system obey the boson commutation relation $\left[a_{j}, a_{j}^{\dagger}\right]=1$. The field at the input could be either in a separable state or in an entangled state. However, the explicit numerical results would be given for a separable though nonclassical input state. The output, generally, would exhibit entanglement.

The Hamiltonian for the system can be written as

$$
H=\hbar g \sum_{j=1}^{N} a_{j}^{\dagger} a_{j}+\hbar J \sum_{j=1}^{N-1}\left(a_{j}^{\dagger} a_{j+1}+a_{j+1}^{\dagger} a_{j}\right) .
$$

In the above equation, $N$ is the number of waveguides and the coupling parameter $J$ represents the rate at which the photons are transferred to the neighboring waveguides. The Hamiltonian (1) can be diagonalized by using the normal coordinates given by

$$
\begin{aligned}
& a_{j}(t)=\sum_{p=1}^{N} b_{p} S(j, p), \\
& b_{p}(t)=\sum_{j=1}^{N} a_{j} S(j, p),
\end{aligned}
$$

where the function $S(j, p)$ is defined as

$$
S(j, p) \equiv \sqrt{\frac{2}{N+1}} \sin \left(\frac{j p \pi}{N+1}\right) .
$$

This function satisfies the orthonormality relations

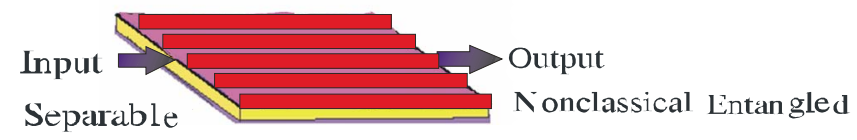

FIG. 1. (Color online) Coupled waveguide array. 


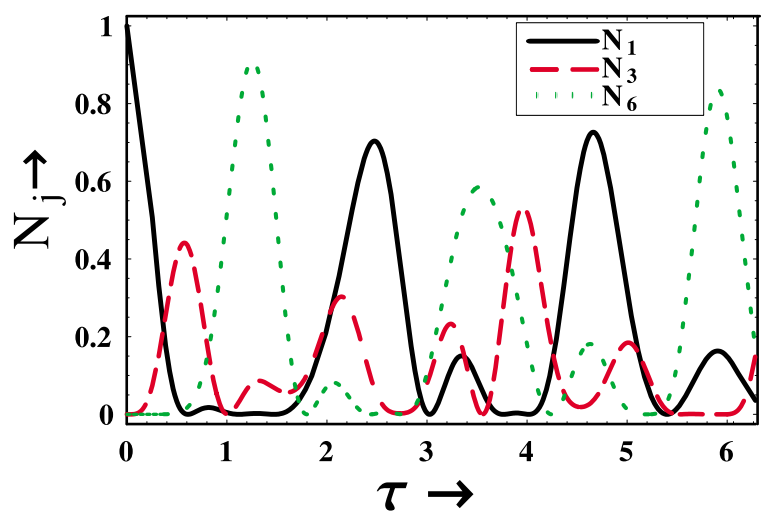

FIG. 2. (Color online) Behavior of the normalized intensity as a function of $\tau(\tau \equiv J t / \pi)$. The input is injected into the first waveguide and the number of waveguides in the system is $N=6$.

$$
\begin{gathered}
\sum_{p=1}^{N} S(n, p) S(m, p)=\delta_{n m}, \\
\sum_{p=1}^{N-1}[S(n, p) S(m, p+1)+S(n, p+1) S(m, p)] \\
=2 \delta_{n m} \cos \left(\frac{n \pi}{N+1}\right) .
\end{gathered}
$$

These two relations lead to the diagonalization of Eq. (1):

$$
\begin{aligned}
& H=\hbar \sum_{p=1}^{N}\left(g+\beta_{p}\right) b_{p}^{\dagger} b_{p}, \\
& \beta_{p} \equiv 2 J \cos \left(\frac{p \pi}{N+1}\right) .
\end{aligned}
$$

Using Eqs. (2)-(6) we obtain the Heisenberg operators for the field in each waveguide [19]

$$
\begin{gathered}
a_{j}(t)=\sum_{l=1}^{N} a_{l}(0) A_{j, l}, \\
A_{j, l} \equiv \sum_{p=1}^{N} \exp \left[-i\left(g+\beta_{p}\right) t\right] S(l, p) S(j, p) .
\end{gathered}
$$

The coefficients $A_{j, l}$ determine all the quantum properties of light in the coupled waveguide system. For example, if we start with light in the $l$ th waveguide then $\left|A_{j, l}\right|^{2}$ gives the propagation of light from the $l$ th to the $j$ th waveguide. The mean number of photons in the $j$ th waveguide would be

$$
N_{j}(t) \equiv N_{l}(0)\left|A_{j, l}(t)\right|^{2} .
$$

This result is similar to the result for classical propagation. Clearly Eq. (8) is independent of $g$. In Fig. 2 we show the normalized intensity when the input is a single photon state. Using the analytical solution (7) we can also build up the wave function of the field at time $t$. The form of the wave function is rather involved. For input Fock states the state at time $t$ can be obtained in terms of the $A_{j, l}$ 's given by Eq. (7):

$$
\left|n_{1}, n_{2}, \ldots, n_{N}\right\rangle \rightarrow \prod_{j} \frac{\left[\Sigma A_{j, l}^{*}(-t) a_{l}^{\dagger}\right]^{n_{j}}}{\sqrt{\left(n_{j}\right) !}}|0,0, \ldots, 0\rangle .
$$

For input single photons in say waveguides $i$ and $j$ the probability of finding one photon in the waveguide $k$ and one in the $l$ th waveguide is $\left|A_{i, k} A_{j, l}+A_{i, l} A_{j, k}\right|^{2}$. The two quantum amplitudes can obviously interfere. In particular let us consider if the coupled waveguides can exhibit an analog of HongOu-Mandel interference. Consider two waveguides with one photon put in each, with a delay of $T$ seconds. The wave function at time $t$ can be related to the initial wave function using the evolution operator $U(t)$ :

$$
|\psi(t)\rangle=\frac{U(t-T) a_{2}^{\dagger} U(T) a_{1}^{\dagger}|0,0\rangle}{\left[\left\langle 1,0\left|U^{\dagger}(T) a_{2} a_{2}^{\dagger} U(T)\right| 1,0\right\rangle\right]^{1 / 2}} .
$$

In Eq. (10) $a_{2}^{\dagger}$ corresponds to the addition of a photon in the second waveguide at time $T$. Further the denominator in Eq. (10) arises as we have to insure the normalization of the wave function $a_{2}^{\dagger} U(T) a_{1}^{\dagger}|0,0\rangle$ at time $T$. Using $U(t-T)$ $=U(t) U^{\dagger}(T)$ and the definition of the Heisenberg operators $a(t)=U^{\dagger}(t) a U(t)$, the numerator in Eq. (10) simplifies to

$$
\begin{aligned}
U(t) a_{2}^{\dagger}(T) a_{1}^{\dagger}|0,0\rangle & =a_{2}^{\dagger}(T-t) a_{1}^{\dagger}(-t) U(t)|0,0\rangle \\
& =a_{2}^{\dagger}(T-t) a_{1}^{\dagger}(-t)|0,0\rangle .
\end{aligned}
$$

Using the solution of Heisenberg equations in this numerator and using Eq. (10) we find that the probability of finding simultaneously one photon at each output at time $t$ is $\left(\theta=J t, \theta_{o}=J T\right)$

$$
\begin{aligned}
p(t, T) & =|\langle 1,1 \mid \psi\rangle|^{2} \\
& =\cos ^{2}\left(2 \theta-\theta_{0}\right) /\left[1+\sin ^{2}\left(\theta_{0}\right)\right],
\end{aligned}
$$

which shows the two photon interference dip at $2 t-T$ $=\pi / 2 \mathrm{~J}$ depending on the length (proportional to $t$ ) of the waveguides and the delay time. For a given structure such a dip can be scanned by varying the delay time.

For initial excitation in a single waveguide the number of photons in each waveguide does not depend on the quantum characteristics of the input field. We therefore examine next the squeezing and entanglement aspects of the radiation in different waveguides. We investigate the propagation of nonclassical light across the coupled waveguides. We assume that squeezed light is coupled into the first waveguide. The input at the first waveguide is

$$
\begin{aligned}
|\zeta\rangle & \equiv \exp \left(-\frac{r}{2}\left[e^{i \phi}\left(a_{1}^{\dagger}\right)^{2}+e^{-i \phi}\left(a_{1}\right)^{2}\right]\right)|0\rangle \\
& \equiv \frac{1}{\sqrt{\cosh r}} \sum_{n=0}^{\infty} \frac{\sqrt{(2 n) !}}{2^{n} n !}[-\exp (i \phi) \tanh (r)]^{n}|2 n\rangle,
\end{aligned}
$$

where $r$ is the magnitude of squeezing and $\phi$ is related to the orientation of the squeezing ellipse. In what follows we set $g=0$. Its effect can always be incorporated by carrying out a simple rotation.

In order to study the transport of inter-waveguide squeezing, we introduce the quadrature operators for the $j$ th waveguide given by $q_{j} \equiv\left(a_{j}+a_{j}^{\dagger}\right) / \sqrt{2}$ and $p_{j} \equiv\left(a_{j}-a_{j}^{\dagger}\right) / \sqrt{2} i$. We 


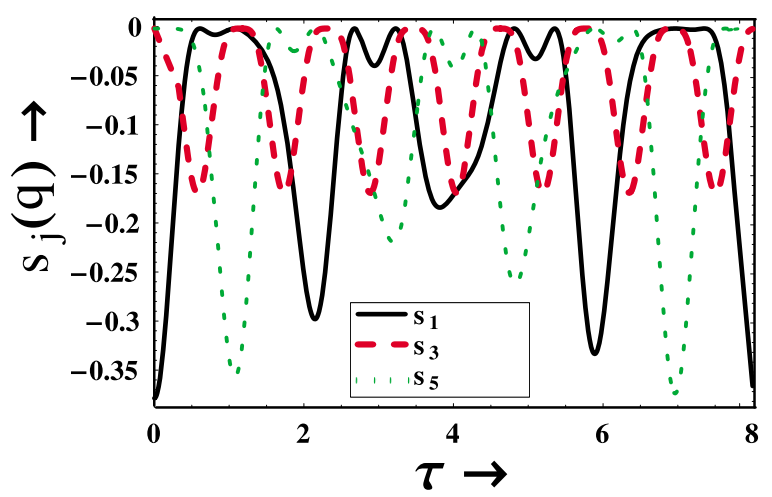

FIG. 3. (Color online) Time evolution of the squeezing factor $s_{j}(q)$ as a function of $\tau(\tau \equiv J t / \pi)$. The input is in the first waveguide and the number of waveguides in the system is $N=5$. The magnitude and the phase of the squeezing parameter are chosen as $r=0.7$ and $\phi=0$, respectively.

also define the squeezing factors $s_{j}(q) \equiv\left(\Delta q_{j}\right)^{2}-1 / 2$ and $s_{j}(p) \equiv\left(\Delta p_{j}\right)^{2}-1 / 2$. Thus squeezing occurs when one of these expressions becomes less than zero. Using Eq. (7) we get
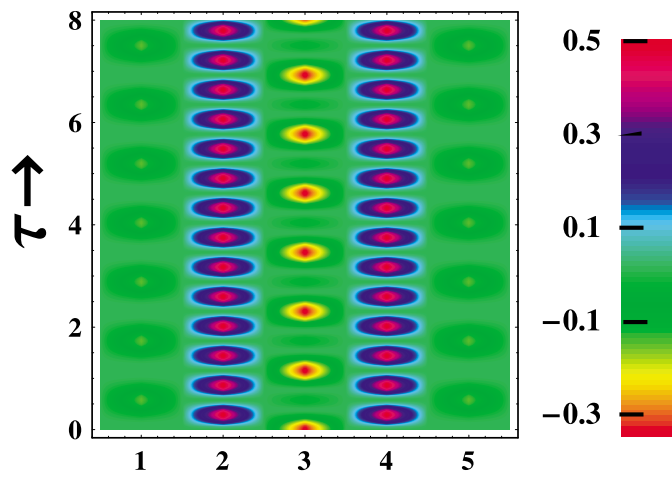

(a)
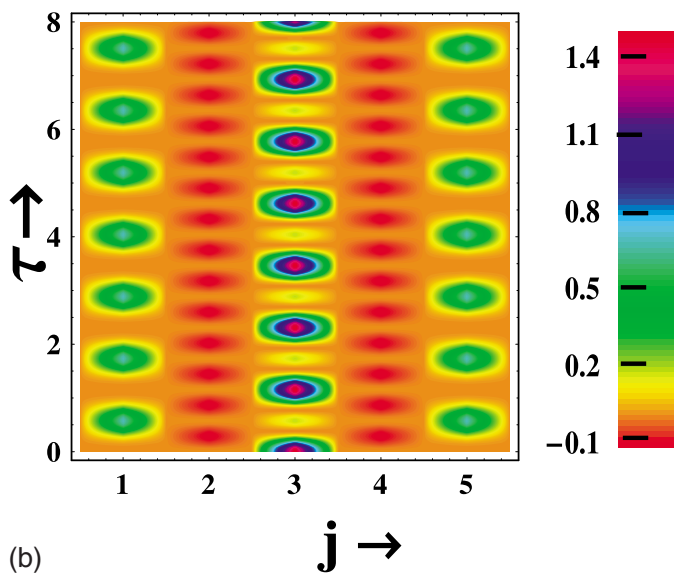

FIG. 4. (Color online) The top [bottom] part shows the variation of $s_{j}(q)\left[s_{j}(p)\right]$ as a function of $\tau \equiv J t / \pi$ for $j=1, \ldots, 5$, smeared out in the $j$ direction. The magnitude and the phase of the squeezing parameter are chosen as $r=0.7$ and $\phi=0$, respectively. The number of waveguides in the system is $N=5$.
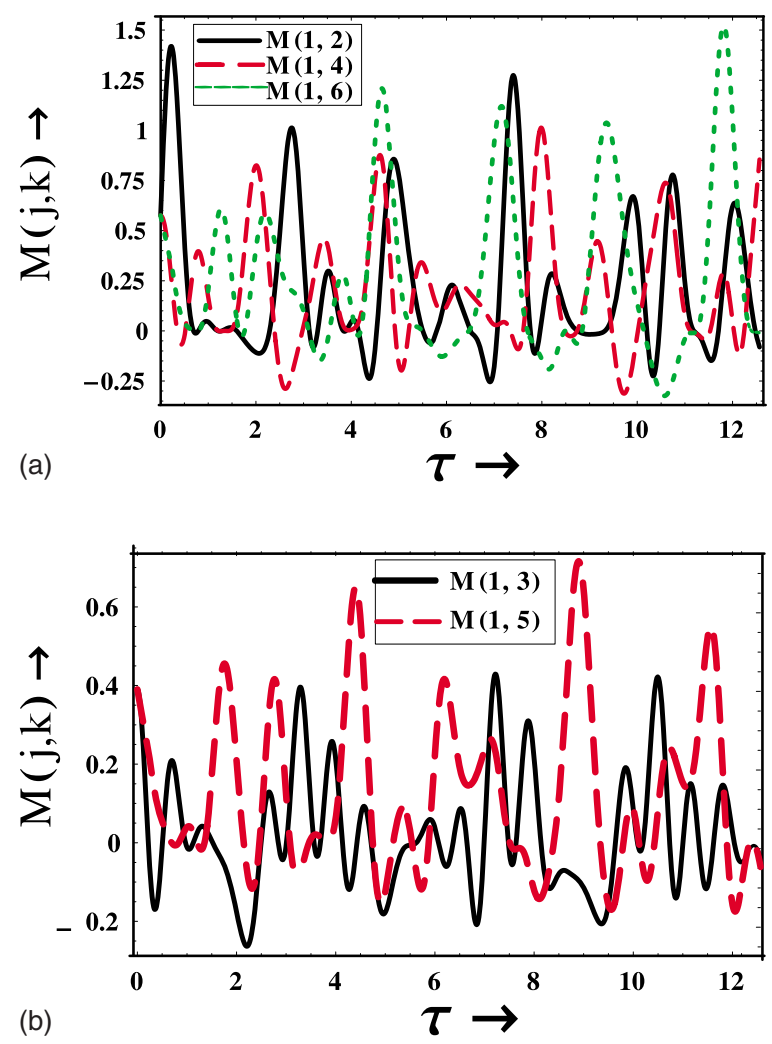

FIG. 5. (Color online) The correlation function $M(j, k)$ as a function of $\tau(\tau \equiv J t / \pi)$ for the case of six waveguides. The magnitude and the phase of the squeezing parameter are chosen as (a) $r=0.7$ and $\phi=3 \pi / 2$ and (b) $r=0.6$ and $\phi=\pi$, respectively.

$$
s_{j}=\left|A_{j, l}\right|^{2} \sinh ^{2} r \mp \frac{1}{4} \sinh 2 r\left[A_{j, l}^{2} \exp (i \phi)+\text { c.c. }\right],
$$

where the $-(+)$ sign is to be used for the quadrature $q(p)$.

In particular for a system of two waveguides, we have

$$
\begin{aligned}
s_{1}(q) & =\cos ^{2}(J t) \sinh (r)[\sinh (r)-\cos (\phi) \cosh (r)] \\
& =s_{2}(p) \cot ^{2}(J t) .
\end{aligned}
$$

Clearly $q$-quadrature is initially squeezed if $\tanh (r)$ $<\cos (\phi)$. Note that for two coupled waveguides we obtain complete transfer of squeezing albeit from $q$-quadrature to $p$-quadrature for $J t=\pi / 2$ [20].

For a system of three waveguides, we get the following results:

$$
\begin{aligned}
& s_{1}(q)=f \cos ^{4}\left(\frac{J t}{\sqrt{2}}\right), \\
& s_{3}(q)=f \sin ^{4}\left(\frac{J t}{\sqrt{2}}\right), \\
& s_{2}(p)=\frac{f}{2} \sin ^{2}(\sqrt{2} J t),
\end{aligned}
$$

$f \equiv \sinh (r)[\sinh (r)-\cos (\phi) \cosh (r)]$. 
The inter-waveguide transfer of squeezing is governed by the factors $\cos ^{4}(J t / \sqrt{2}), \quad \sin ^{4}(J t / \sqrt{2})$, and $2 \sin ^{2}(J t /$ $\sqrt{2}) \cos ^{2}(J t / \sqrt{2})$. Again, the $q$-quadrature is initially squeezed if $\tanh (r)<\cos (\phi)$. Also, for the case of three coupled waveguides we obtain complete transfer of squeezing from the first waveguide to the third waveguide for $J t=\pi / \sqrt{2}$. In Fig. 3, we display the time evolution of the quadrature squeezing for the case of five waveguides. The negative values of $s_{q}(1), s_{q}(3)$, and $s_{q}(5)$ clearly demonstrate the squeezing in the $q$-quadrature. Figure 4 shows the quadrature squeezing when the input is given to the middle waveguide.

We next show that input of nonclassical light to one of the waveguides can produce pairwise entanglement between the inter-waveguide modes. We use the well-known criterion for entanglement between two continuous variable systems [21-23]. As a measure of entanglement we examine the correlation between two waveguide modes.

$$
M(j, k)=\left\langle a_{j}^{\dagger} a_{j}\right\rangle+\left\langle a_{k}^{\dagger} a_{k}\right\rangle+\left\langle a_{j} a_{k}\right\rangle+\left\langle a_{j}^{\dagger} a_{k}^{\dagger}\right\rangle .
$$

The negativity of $M$ is a sufficient condition for entanglement. For Gaussian states this is both necessary and sufficient. A calculation shows that the joint state of the coupled waveguides is Gaussian. We calculate $M$ using the solution (8) and the state in Eq. (12). Before we show numerical results, we discuss analytical results for two and three waveguides. In particular, for a system of two waveguides,

$$
M(1,2)=\frac{1}{2}\{\sinh (2 r)[\tanh r-\sin (2 J t) \sin \phi]\}
$$

and thus entanglement occurs for $\sin (2 J t) \sin (\phi)>\tanh (r)$.
For a system of three waveguides, we find the results

$$
\begin{aligned}
M(1,2)= & \frac{1}{2} \cos ^{2}\left(\frac{J t}{\sqrt{2}}\right)\left\{[3-\cos (\sqrt{2} J t)] \sinh ^{2}(r)\right. \\
& -\sqrt{2} \sin (\phi) \sinh (2 r) \sin (\sqrt{2} J t)\}, \\
M(1,3)= & \frac{1}{4}\left\{\cos (\phi) \sinh (2 r) \sin ^{2}(\sqrt{2} J t)\right. \\
& \left.+[3+\cos (2 \sqrt{2} J t)] \sinh ^{2}(r)\right\} .
\end{aligned}
$$

Clearly for $\phi=\pi$, the first and third could be entangled. In Fig. 5, we show the time evolution of $M(j, k)$ for the case of six waveguides. The negative values of $M(1, j)(j=1-6)$ in Figs. 5(a) and 5(b) clearly demonstrate the entanglement between the inter-waveguide modes.

In conclusion, we discussed the continuous time quantum walk of quantum particles (photons) in a physical system consisting of $N$ coupled waveguides. We showed that the quantum walk with a light source having strong quantum character can produce entanglement between different waveguides in the array. We also studied the transport of nonclassical light across the coupled waveguides. We could investigate several other interesting nonclassical situations, for example, the effect of launching a distribution of entangled photon pairs into the array [24]. One could also ask: If the input wave function for photons is periodic, then how is this periodicity reflected at the output wave function? This would provide us with an analogy of the Talbot effect in the second quantized setup.
[1] P. A. M. Dirac, The Principles of Quantum Mechanics (Oxford University Press, New York, 1958).

[2] C. K. Hong, Z. Y. Ou, and L. Mandel, Phys. Rev. Lett. 59, 2044 (1987).

[3] H. Huang and G. S. Agarwal, Phys. Rev. A 49, 52 (1994).

[4] M. S. Kim, W. Son, V. Bužek, and P. L. Knight, Phys. Rev. A 65, 032323 (2002); W. K. Lai, V. Bužek, and P. L. Knight, ibid. 43, 6323 (1991).

[5] B. H. Liu, F. W. Sun, Y. X. Gong, Y. F. Huang, G. C. Guo, and Z. Y. Ou, Opt. Lett. 32, 1320 (2007).

[6] H. Wang and T. Kobayashi, Phys. Rev. A 71, 021802(R) (2005).

[7] T. Nagata, R. Okamoto, J. L. O’Brien, K. Sasaki, and S. Takeuchi, Science 316, 726 (2007).

[8] M. Paternostro, H. McAneney, and M. S. Kim, Phys. Rev. Lett. 94, 070501 (2005).

[9] R. Iwanow, D. A. May-Arrioja, D. N. Christodoulides, G. I. Stegeman, Y. Min, and W. Sohler, Phys. Rev. Lett. 95, 053902 (2005).

[10] H. B. Perets, Y. Lahini, F. Pozzi, M. Sorel, R. Morandotti, and Y. Silberberg, Phys. Rev. Lett. 100, 170506 (2008).

[11] R. P. Feynman and A. R. Hibbs, Quantum Mechanics and Path Integrals (McGraw-Hill, New York, 1965).
[12] Y. Aharonov, L. Davidovich, and N. Zagury, Phys. Rev. A 48, 1687 (1993).

[13] Y. Omar, N. Paunković, L. Sheridan, and S. Bose, Phys. Rev. A 74, 042304 (2006).

[14] H. Jeong, M. Paternostro, and M. S. Kim, Phys. Rev. A 69, 012310 (2004).

[15] P. K. Pathak and G. S. Agarwal, Phys. Rev. A 75, 032351 (2007).

[16] B. Do, M. L. Stohler, S. Balasubramanian, D. S. Elliott, C. Eash, E. Fischbach, M. A. Fischbach, A. Mills, and B. Zwickl, J. Opt. Soc. Am. B 22, 499 (2005).

[17] A. Biswas and G. S. Agarwal, Phys. Rev. A 70, 022323 (2004); M. A. Sillanpää, J. I. Park, and R. W. Simmonds, Nature (London) 449, 438 (2007).

[18] A recent paper, A. Politi, M. J. Cryan, J. G. Rarity, S. Yu, and J. L. O'Brien, Science 320, 646 (2008), discusses the application of waveguides to quantum circuits.

[19] For general results on the generated quantum states for the case of linear transformations see [3]; [8]; and S. L. Braunstein, Phys. Rev. A 71, 055801 (2005).

[20] Transfer of quantum correlations from one frequency to the other was studied by J. Huang and P. Kumar, Phys. Rev. Lett. 68, 2153 (1992). 
[21] L.-M. Duan, G. Giedke, J. I. Cirac, and P. Zoller, Phys. Rev. Lett. 84, 2722 (2000).

[22] R. Simon, Phys. Rev. Lett. 84, 2726 (2000).

[23] S. Mancini, V. Giovannetti, D. Vitali, and P. Tombesi, Phys. Rev. Lett. 88, 120401 (2002).
[24] A recent paper, Y. Bromberg, Y. Lahini, R. Morandotti, and Y. Silberberg, e-print arXiv:0807.3938, calculates the photonphoton correlations at the output port when two photons either in a factorized state or in an entangled state are sent in at two different ports. 